\title{
Outburst of GX 304-1 monitored with INTEGRAL: positive correlation between the cyclotron line energy and flux
}

\author{
D. Klochkov ${ }^{1}$, V. Doroshenko ${ }^{1}$, A. Santangelo ${ }^{1}$, R. Staubert ${ }^{1}$, C. Ferrigno ${ }^{2}$, P. Kretschmar ${ }^{3}$, I. Caballero ${ }^{4}$, J. Wilms ${ }^{5}$,
} I. Kreykenbohm ${ }^{5}$, K. Pottschmidt ${ }^{6,9}$, R. E. Rothschild ${ }^{7}$, C. A. Wilson-Hodge ${ }^{8}$, and G. Pühlhofer ${ }^{1}$

${ }^{1}$ Institut für Astronomie und Astrophysik, Universität Tübingen (IAAT), Sand 1, 72076 Tübingen, Germany e-mail: klochkov@astro.uni-tuebingen.de

2 ISDC Data Center for Astrophysics, University of Geneva, chemin d'Écogia 16, 1290 Versoix, Switzerland

3 European Space Astronomy Centre (ESA/ESAC), Science Operations Department, Villanueva de la Canãda, Madrid, Spain

${ }^{4}$ CEA Saclay, DSM/IRFU/SAp - UMR AIM (7158) CNRS/CEA/Université P. Diderot, Orme des Merisiers, Bât. 709, 91191 Gif-sur-Yvette, France

5 Dr. Karl Remeis Sternwarte \& Erlangen Centre for Astroparticle Physics, Sternwartstr. 7, 96049 Bamberg, Germany

${ }^{6}$ Center for Space Science and Technology, University of Maryland Baltimore County, 1000 Hilltop Circle, Baltimore, MD 21250 , USA

7 Center for Astrophysics and Space Sciences, University of California, San Diego, 9500 Gilman Dr., La Jolla, CA 92093-0424, USA

${ }^{8}$ NASA Marshall Space Flight Center, Huntsville, AL 35812, USA

9 CRESST \& NASA Goddard Space Flight Center, Astrophysics Science Division, Code 661, Greenbelt, MD 20771, USA

Received 11 April 2012 / Accepted 23 May 2012

\section{ABSTRACT}

Context. X-ray spectra of many accreting pulsars exhibit significant variations as a function of flux and thus of mass accretion rate. In some of these pulsars, the centroid energy of the cyclotron line(s), which characterizes the magnetic field strength at the site of the $\mathrm{X}$-ray emission, has been found to vary systematically with flux.

Aims. GX 304-1 is a recently established cyclotron line source with a line energy around $50 \mathrm{keV}$. Since 2009, the pulsar shows regular outbursts with the peak flux exceeding one Crab. We analyze the INTEGRAL observations of the source during its outburst in January-February 2012.

Methods. The observations covered almost the entire outburst, allowing us to measure the source's broad-band X-ray spectrum at different flux levels. We report on the variations in the spectral parameters with luminosity and focus on the variations in the cyclotron line.

Results. The centroid energy of the line is found to be positively correlated with the luminosity. We interpret this result as a manifestation of the local sub-Eddington (sub-critical) accretion regime operating in the source.

Key words. X-rays: binaries - stars: neutron - accretion, accretion disks

\section{Introduction}

In accreting binary pulsars, matter from the normal stellar companion is transferred to a highly magnetized $\left(B \gtrsim 10^{12} \mathrm{G}\right)$ neutron star. In the vicinity of the accretor, the gas flow is disrupted by the neutron star's magnetic field and channeled towards the magnetic poles, where most of the X-rays originate. The physics and the structure of the X-ray emitting region(s) above the neutron star surface are still highly debated (see, e.g., Becker \& Wolff 2007; Farinelli et al. 2012). Since the matter hitting the accretor's surface is highly ionized, the magnetic field strength is a crucial parameter determining the physical processes inside the emitting region and the formation of the observed X-ray spectrum. A direct way to assess the $B$-field strength at the site of X-ray emission is the measurement of the cyclotron resonant scattering features (CRSF or cyclotron lines) in the X-ray spectrum of a pulsar. These features appear as absorption lines, caused by the resonant scattering of photons off the electrons in Landau levels (see, e.g., Trümper et al. 1978; Isenberg et al. 1998). The energy of the fundamental line and the spacing between the harmonics are directly proportional to the field strength.
In some accreting pulsars, the energy of the cyclotron line has been found to vary with luminosity, apparently due to a displacement of the line formation region. Such variations of the line energy have been reported for V $0332+53$ (e.g., Tsygankov et al. 2010), 4U 0115+63 (e.g., Tsygankov et al. 2010), Her X-1 (Staubert et al. 2007; Klochkov et al. 2011), and A 0535+26 (Klochkov et al. 2011). The luminosity-dependence of the cyclotron feature has strong implications for the physics of the $\mathrm{X}$-ray emitting region as discussed, e.g., by Staubert et al. (2007), Klochkov et al. (2011), and Becker \& Wolff (2007).

GX 304-1 is a recently established cyclotron line source (Yamamoto et al. 2011). It was discovered in a balloon experiment in 1967 and subsequently identified as an X-ray pulsar with a period of $\sim 272 \mathrm{~s}$ (McClintock et al. 1977). The system contains a Be-type optical companion (Mason et al. 1978) and is located at a distance of $2.4 \mathrm{kpc}$ (Parkes et al. 1980). Since 1980, GX 304-1 has remained in a quiescent state, showing no outbursts. Starting from 2008, when the source was detected with INTEGRAL (Manousakis et al. 2008), GX 304-1 "resumed" its activity exhibiting outbursts with a period of $\sim 132.5 \mathrm{~d}$.

The energy of the cyclotron line in GX 304-1 was measured with Suzaku and RXTE to be around $52 \mathrm{keV}$ by 
Table 1. INTEGRAL observations of the GX304-1 outburst in January-February 2012.

\begin{tabular}{cccccc}
\hline \hline \multirow{2}{*}{ Rev. } & Obs. ID & Mid. MJD & \multicolumn{3}{c}{ Exposure [ks] } \\
& & & JEM-X & IBIS & SPI \\
\hline 1131 & 09400230006 & 55944.0 & 64.6 & 42.7 & 68.6 \\
1132 & 09400230007 & 55947.0 & 42.4 & 31.9 & 36.6 \\
1133 & 09400230008 & 55950.0 & - & - & 10.7 \\
1134 & 09400230009 & 55952.8 & 7.3 & 25.4 & 37.8 \\
1135 & 09400230010 & 55955.7 & - & 6.7 & 25.1 \\
1136 & 09400230011 & 55958.7 & 36.9 & 28.1 & 32.9 \\
1137 & 09400230012 & 55962.0 & 78.1 & 59.7 & 78.4 \\
1138 & 09400230013 & 55965.0 & 60.7 & 45.2 & 52.3 \\
\hline
\end{tabular}

Yamamoto et al. (2011). These authors demonstrate that the data taken at different flux levels show an indication of a positive correlation between the line energy and the X-ray flux, although at a low significance level. This made GX 304-1 a good target for a luminosity-dependent study of the cyclotron feature. In this work, we present the analysis of INTEGRAL data (see next section for the mission description) taken during the January 2012 outburst of the source. The data reveal a positive correlation between the cyclotron line energy and the source flux, as well as the variation in other spectral parameters during the outburst. We discuss our finding in the context of a model assuming that different accretion regimes can operate in a particular pulsar depending on its X-ray luminosity.

\section{Observations and data reduction}

At the beginning of January 2012, GX 304-1 entered an outburst as reported by Yamamoto et al. (2012). The outburst was monitored by the International Gamma-Ray Astrophysics Laboratory - INTEGRAL (Winkler et al. 2003), starting at MJD 55 943.5, when the source flux in the $\sim 20-80 \mathrm{keV}$ range was $\sim 250 \mathrm{mCrab}$, through the maximum of the outburst, when the source flux exceeded one Crab, to MJD 55 965.5, when the flux dropped to $\sim 100$ mCrab. INTEGRAL performed one observation every satellite orbit (about three days), with a typical exposure of a few tens of kiloseconds each. In total, eight observations were performed. The INTEGRAL scientific payload contains three X-ray instruments: (i) the imager IBIS sensitive from $\sim 20 \mathrm{keV}$ to a few MeV (Ubertini et al. 2003; Lebrun et al. 2003); (ii) the spectrometer SPI sensitive in roughly the same energy range as IBIS (Vedrenne et al. 2003); and (iii) the X-ray monitor JEM-X operating between $\sim 3$ and $\sim 35 \mathrm{keV}$ (Lund et al. 2003). Table 1 summarizes the INTEGRAL observations of GX 304-1. The increased solar activity during the observations led to the reduction in the exposure time and availability of the instruments as can be seen from the exposure columns of Table 1.

The INTEGRAL observations are indicated in Fig. 1, which shows the Swift/BAT light curve of GX304-1 ${ }^{1}$. The INTEGRAL monitoring has an excellent coverage of the entire outburst, providing a rare opportunity to follow the evolution of the outburst from the early rising phase to the late decay phase.

For our analysis, we used data from the ISGRI detector of IBIS, which is sensitive in the 20-300 keV energy range, JEM-X, and SPI. Standard data processing was performed with version 9 of the Offline Science Analysis (OSA) software. We performed an additional gain correction of the

\footnotetext{
1 We used the Swift/BAT transient monitor results provided by the Swift/BAT team.
}

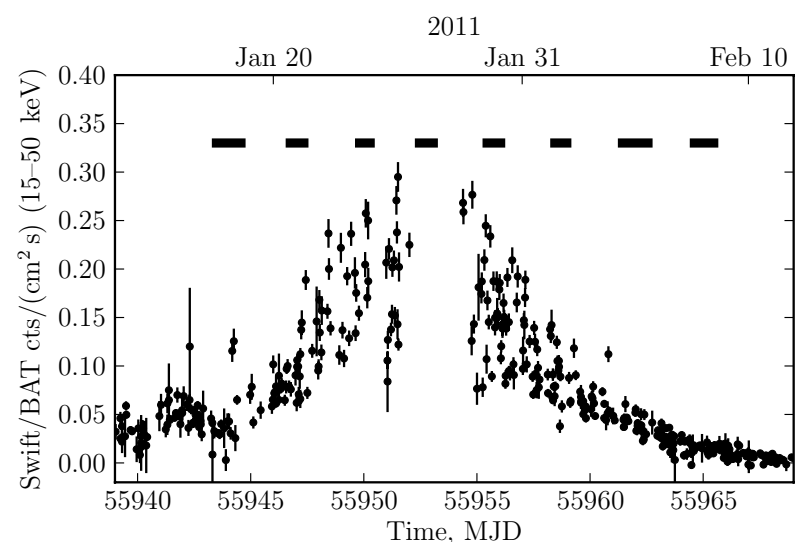

Fig. 1. INTEGRAL observations of GX 304-1 (horizontal bars) superposed on the Swift/BAT light curve of the source during its outburst in January-February 2012 ( $\sim 0.22$ units of the vertical axis corresponds to one Crab).

ISGRI energy scale based on the background spectral lines of Tungsten (the modified response files based on the nearest Crab observations were used).

\section{Spectral analysis}

Figure 1 shows that the X-ray flux of GX 304-1 during the observed part of the outburst changed by an order of magnitude. This allowed a detailed study of the luminosity dependence of the source's broad-band X-ray spectrum. X-ray pulsations with a period of $\sim 274.9 \mathrm{~s}$ were detected in all INTEGRAL observations. This value is roughly consistent with the known pulse period. A detailed timing analysis is not part of the present work and will be presented elsewhere.

In all observations, the X-ray continuum could be closely modeled by a standard power-law/cutoff function (flux $\propto E^{-\Gamma} \exp \left[E / E_{\text {fold }}\right]$, where $E$ is the photon energy, $\Gamma$ is the photon index, and $E_{\mathrm{fold}}$ is the exponential roll-off parameter) modified by photo-electric absorption at low energies. In addition, the spectra showed a cyclotron resonant scattering feature around $\sim 50 \mathrm{keV}$ in absorption, which was modeled with a multiplicative absorption line with a Gaussian optical depth profile $I(E)=I_{\text {cont }}(E) \cdot \mathrm{e}^{-G(E)}$, where $G(E)=-\tau_{\text {cyc }} /\left(\sqrt{2 \pi} \sigma_{\text {cyc }}\right)$. $\exp \left[-0.5\left(E-E_{\text {cyc }}\right)^{2} / \sigma_{\text {cyc }}^{2}\right], I_{\text {cont }}(E)$ is the continuum function, $E_{\text {cyc }}, \sigma_{\text {cyc }}$, and $\tau_{\text {cyc }}$ are the centroid energy, width, and optical depth of the line, respectively. The line is clearly detected in ISGRI and SPI data separately, as shown by the residual plots in Fig. 2. The inclusion of the absorption line in the model leads to an improvement in the reduced $\chi^{2}$ from 3.10 for 145 d.o.f. (with the corresponding null-hypothesis probability of only $\sim 10^{-32}$ ) to 0.75 for 142 d.o.f. (null-hypothesis probability $>0.9$ ). The energy of the line is consistent with that reported by Yamamoto et al. (2011) based on the RXTE and Suzaku observations. We also included an additive Gaussian component to model the $\mathrm{Fe} K_{\alpha}$ fluorescence emission line around $6.4 \mathrm{keV}$. The inclusion of the line reduces the residuals around $6 \mathrm{keV}$. The corresponding improvement of the reduced $\chi^{2}$ is, however, marginal: from 0.80 (143 d.o.f.) to 0.75 (142 d.o.f.). The presence of the Fe line is, therefore, questionable.

Both the continuum and cyclotron line parameters vary systematically during the outburst. Here, we focus on the evolution of the cyclotron line energy $E_{\text {cyc }}$, to establish a possible correlation of $E_{\mathrm{cyc}}$ with flux, similar to that found by Yamamoto et al. (2011). To characterize this variability, we used 
D. Klochkov et al.: Cyclotron line in GX 304-1 correlates positively with flux

Table 2. Best-fit spectral parameters with the corresponding $1 \sigma$-uncertainties for the INTEGRAL observations used in this work.

\begin{tabular}{lcccccc}
\hline \hline Revolution & 1131 & 1132 & 1134 & 1136 & 1137 & 1138 \\
\hline$\Gamma$ & $1.10_{-0.03}^{+0.03}$ & $0.83_{-0.03}^{+0.03}$ & $0.93_{-0.09}^{+0.09}$ & $1.10_{-0.08}^{+0.08}$ & $1.23_{-0.08}^{+0.08}$ & $1.56_{-0.05}^{+0.13}$ \\
$E_{\text {fold }}, \mathrm{keV}$ & $19.1_{-0.6}^{+0.7}$ & $15.5_{-0.4}^{+0.4}$ & $14.5_{-0.6}^{+0.6}$ & $16.9_{-0.7}^{+0.8}$ & $19.5_{-1.0}^{+1.1}$ & $28.6_{-2.2}^{+3.8}$ \\
$E_{\text {cyc }}, \mathrm{keV}$ & $52.98_{-0.75}^{+0.80}$ & $54.39_{-0.73}^{+0.75}$ & $55.28_{-0.81}^{+0.87}$ & $51.44_{-0.52}^{+0.54}$ & $51.70_{-0.63}^{+0.66}$ & $48.37_{-1.12}^{+1.19}$ \\
$\sigma_{\text {cyc }}, \mathrm{keV}$ & $6.45_{-0.58}^{+0.62}$ & $7.56_{-0.57}^{+0.59}$ & $8.45_{-0.75}^{+0.83}$ & $5.60_{-0.46}^{+0.48}$ & $5.44_{-0.55}^{+0.56}$ & $4.80_{-0.89}^{+0.91}$ \\
$\tau_{\text {cyc }}$ & $9.4_{-1.2}^{+1.3}$ & $12.1_{-1.3}^{+1.4}$ & $13.3_{-2.0}^{+2.4}$ & $8.8_{-0.9}^{+0.9}$ & $8.9_{-1.1}^{+1.2}$ & $5.7_{-1.3}^{+1.3}$ \\
Flux $/ 10^{-8} \mathrm{erg} \mathrm{s}^{-1} \mathrm{~cm}^{-2}$ & $0.375_{-0.002}^{+0.002}$ & $0.811_{-0.004}^{+0.004}$ & $1.630_{-0.011}^{+0.011}$ & $0.729_{-0.005}^{+0.005}$ & $0.340_{-0.003}^{+0.002}$ & $0.151_{-0.002}^{+0.002}$ \\
$L_{\mathrm{X}} / 10^{37} \mathrm{erg} \mathrm{s}^{-1}$ & 0.26 & 0.56 & 1.12 & 0.50 & 0.23 & 0.10 \\
$\chi_{\text {red }}^{2} /$ d.o.f. & $1.1 / 164$ & $0.7 / 164$ & $1.3 / 164$ & $1.1 / 164$ & $0.9 / 164$ & $1.0 / 164$ \\
\hline
\end{tabular}

Notes. The luminosity is provided assuming a distance of $2.4 \mathrm{kpc}$ and an isotropic emission diagram.

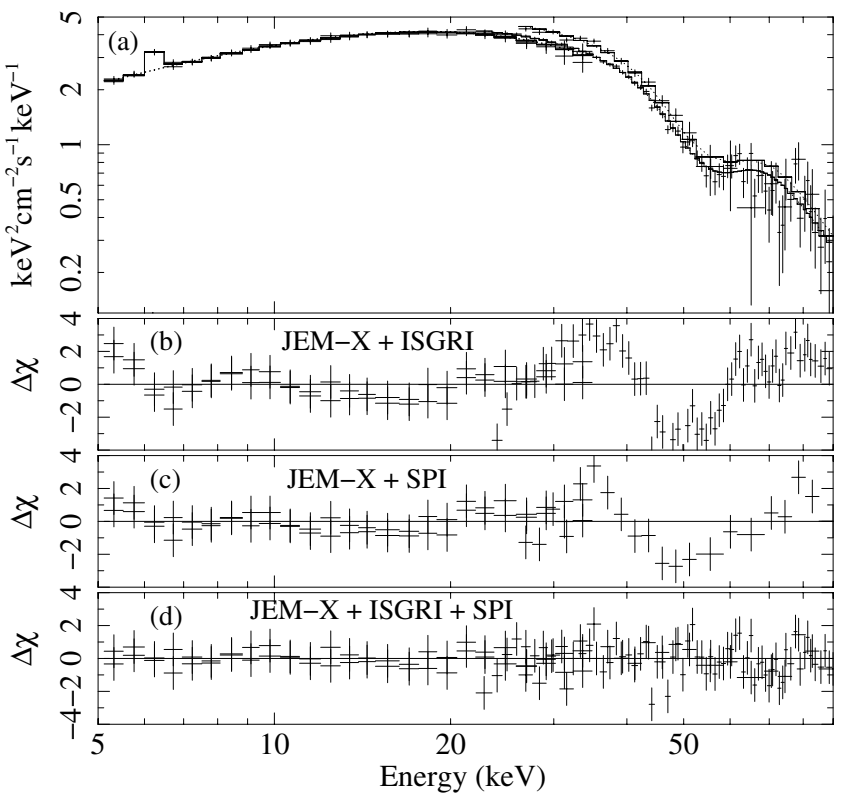

Fig. 2. Example of the INTEGRAL spectrum of GX 304-1 (revolution 1132) with a simultaneous fit of data from JEM-X, ISGRI, and SPI with a power-law/cutoff model including a cyclotron absorption line (see text) a); the residuals for a fit with the model without the cyclotron line for JEM-X + ISGRI b); and JEM-X + SPI c); and with the model where the line is included for data from all instruments $\mathbf{d}$ ).

only observations where data from all three INTEGRAL X-ray instruments were available. This filtering allowed us to obtain a homogeneous set of data and, hence minimize possible systematic effects. Following this selection, data from the orbits 1133 and 1135 were excluded. The JEM-X data collected during the orbit 1134 (peak of the outburst) have been flagged as "bad" by the instrument team owing to the impossibility to provide a precise energy calibration. In our analysis, however, we use the JEM-X data to restrict the low-energy part of the broadband X-ray continuum of the source, for which a precise energy scale is not very critical. Therefore, after consulting the JEM-X team, we re-introduced the JEM-X data from the revolution 1134 in our analysis. We checked, however, that inclusion of the JEM-X data of rev. 1134 to the corresponding spectral fit does not significantly influence the measured energy of the cyclotron line (the focus of this work), but only affects its uncertainty. Table 2 summarizes the best-fit spectral parameters achieved in each observations.

A clear systematic variation in the line energy over the outburst is evident in Fig. 3, which shows that $E_{\text {cyc }}$ generally follows

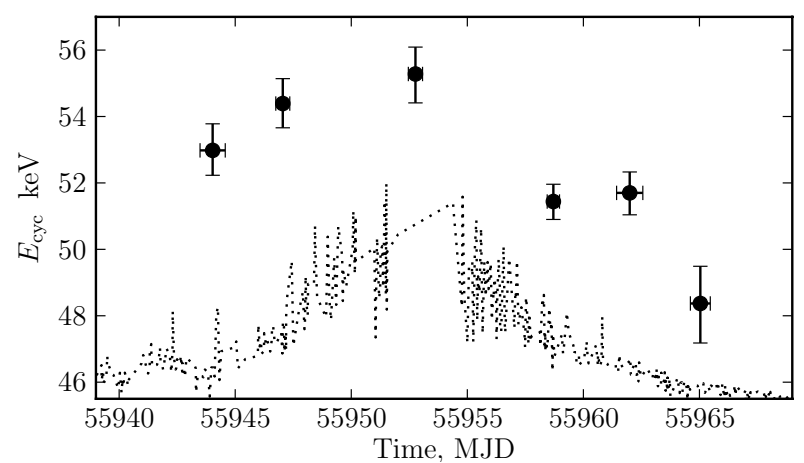

Fig. 3. Evolution of the cyclotron line centroid energy $E_{\text {cyc }}$ throughout the outburst of GX 304-1 as measured with INTEGRAL. The vertical error bars indicate $1 \sigma$-uncertainties. The horizontal error bars indicate the time intervals of the observations. The dotted line represents the re-scaled Swift/BAT light curve.

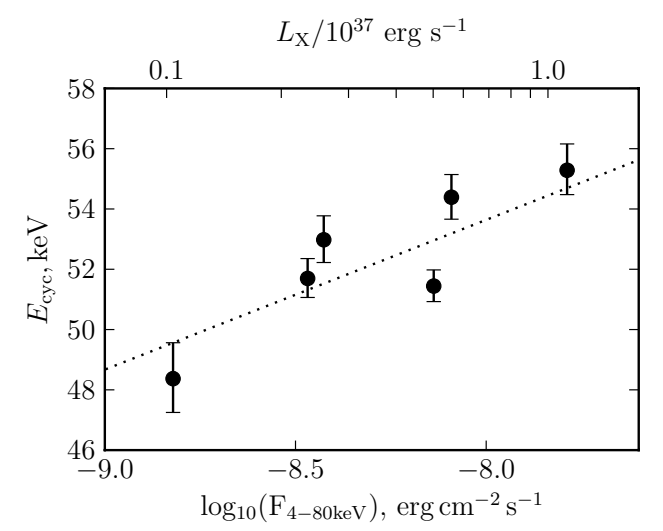

Fig. 4. Cyclotron line centroid energy $E_{\mathrm{cyc}}$ as a function of the logarithm of flux in the $4-80 \mathrm{keV}$ range. The error bars indicate $1 \sigma$-uncertainties (the flux uncertainties are smaller than the symbol size). The top $X$-axis represents the corresponding isotropic source luminosity assuming a distance of $2.4 \mathrm{kpc}$. The dotted line shows a linear fit to the $E_{\text {cyc }}-\log _{10}$ (Flux) dependence.

the X-ray flux. To assess the interdependence of the two parameters, we plotted $E_{\text {cyc }}$ as a function of the X-ray flux in the 4-80 keV range measured with INTEGRAL in the respective observations (Fig. 4). The plot shows a positive correlation between the two values. A linear fit to the dependence of $E_{\text {cyc }}$ on the logarithm of flux (dotted line in the plot) reveals a slope of $4.97 \pm 1.12 \mathrm{keV} / \log _{10}\left(\mathrm{erg} \mathrm{cm}^{-2} \mathrm{~s}^{-1}\right)$. The standard linear correlation analysis of the $E_{\text {cyc }}-\log _{10}$ (Flux) dependence yields a 


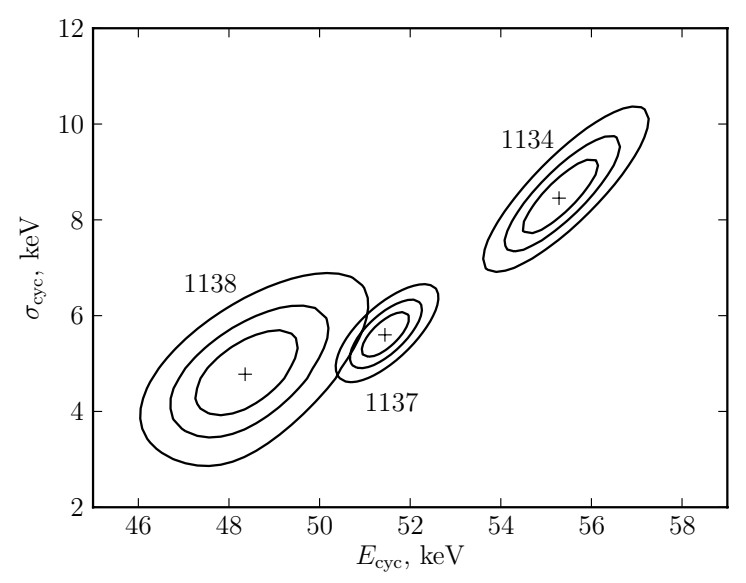

Fig. 5. $\chi^{2}$-contour plots of the parameter pair $E_{\text {cyc }} / \sigma_{\text {cyc }}$ for a few selected observations. The contours correspond to $\chi_{\min }^{2}+1.0$ (the projections of this contour to the parameter axes correspond to the $68 \%$-uncertainty for one parameter of interest), $\chi_{\min }^{2}+2.3$ (68\%-uncertainty for two parameters of interest), and $\chi_{\min }^{2}+4.61$ (90\%-uncertainty for two parameters of interest). The respective orbit numbers are indicated.

Pearson's correlation coefficient of 0.88 with a probability of obtaining the correlation by chance of $\sim 0.01$ (one-sided).

We verified whether our spectral fits contain artificial (model-driven) dependences of the cyclotron line energy $E_{\text {cyc }}$ and other model parameters using $\chi^{2}$-contour plots. No significant model-driven dependences between $E_{\text {cyc }}$ and any of the continuum parameters were found. The line energy was, however, found to be somewhat coupled to the line width $\sigma_{\text {cyc }}$ and its central optical depth $\tau_{\text {cyc }}$. Nevertheless, the $\chi^{2}$-minima and the confidence intervals could be clearly identified and are separated for the different observations as shown in Fig. 5. The plot shows the contours for the $E_{\text {cyc }} / \sigma_{\text {cyc }}$ pair. The $E_{\text {cyc }} / \tau_{\text {cyc }}$ contours look similar.

To check whether the $E_{\text {cyc }} /$ flux correlation is related to instrumental effects, we performed spectral fits using only the JEM-X and SPI data (excluding ISGRI). To verify whether the correlation depends on the choice of the spectral model, we fit the data using a Lorentzian line profile instead of a Gaussian one. We also tried two alternative continuum functions: XSPEC powerlaw $\times$ highecut and compTT models. In the former model, the highecut component controls the exponential roll-off. In addition to $E_{\text {fold }}$, this component has an additional parameter - the cutoff energy $E_{\text {cutoff }}$, above which the spectrum is affected by the roll-off. In our fits, $E_{\text {cutoff }}$ stays between a few and $\sim 10 \mathrm{keV}$. In all cases, the positive $E_{\text {cyc }} /$ flux correlation was reproduced. We conclude, therefore, that the reported correlation arises from the source's behavior and reflects real physics.

\section{Discussion and conclusions}

The positive correlation between the cyclotron line centroid energy and the flux found with INTEGRAL confirms the claim of this correlation by Yamamoto et al. (2011). The revealed dependence establishes GX 304-1 as the third member of a slowly emerging class of accreting pulsars showing a positive $E_{\text {cyc }} /$ flux correlation with the other members being Her X-1 (Staubert et al. 2007) and possibly A $0535+26$ (Klochkov et al. 2011), for which the positive correlation has so far been only established in the pulse-to-pulse analysis. The opposite (negative) correlation between the line energy and the flux was found in V $0332+53$ and 4U 0115+63 (e.g., Tsygankov et al. 2007, 2010). According to discussions in Staubert et al. (2007), Klochkov et al. (2011), and Becker et al. (2012), these two types of dependences reflect two different regimes of accretion. A particular regime is realized in a source depending on whether its X-ray luminosity $L$ is above or below a critical luminosity $L_{\mathrm{c}}$, which corresponds to the local Eddington luminosity at the X-ray emitting structure(s) on/above the neutron star surface. In accreting pulsars radiating above $L_{\mathrm{c}}$ ("super-critical" sources), infalling matter is decelerated in a radiative shock, whose height is believed to increase with $L$, i.e., drift towards an area with a lower $B$-field strength. The opposite behavior probably occurs in sources radiating below or close to $L_{\mathrm{c}}$ ("sub-critical" sources), where infalling matter is stopped by the Coulomb drag and collective plasma effects rather than in a radiative shock. As discussed in Staubert et al. (2007) and Becker et al. (2012), the height of the emitting region decreases with increasing luminosity owing to a corresponding increase in ram pressure of the infalling material, leading to a positive $E_{\text {cyc }} /$ flux correlation. The critical luminosity $L_{\mathrm{c}}$ depends on the parameters of the accreting neutron star, but is generally around a few times $\sim 10^{37} \mathrm{erg} \mathrm{s}^{-1}$ (Basko \& Sunyaev 1976; Staubert et al. 2007; Becker et al. 2012). Assuming a distance of $2.4 \mathrm{kpc}$ (Parkes et al. 1980), the X-ray luminosity of GX 304-1 in the 4-80 keV range during the reported INTEGRAL observations varies between $\sim 1.1 \times$ $10^{36} \mathrm{erg} \mathrm{s}^{-1}$ and $\sim 1.13 \times 10^{37} \mathrm{erg} \mathrm{s}^{-1}$. Thus, according to the described picture, the source should belong to the class of "subcritical" sources, for which a positive $E_{\text {cyc }} /$ flux correlation is expected. The reported observations are, therefore, in agreement with the idea of two accretion regimes and increases the yet very small sample of accreting pulsars with established $E_{\text {cyc }} /$ flux correlations.

Acknowledgements. The work was supported by the Carl-Zeiss-Stiftung. J.W. and I.K. were partially supported by BMWi under DLR grant 50 OR 1007 . This research is based on observations with INTEGRAL, an ESA project with instruments and science data centre funded by ESA member states. We thank the INTEGRAL team for the prompt scheduling of the TOO observations and support with the data reduction and calibration. We thank ISSI (Bern, Switzerland) for its hospitality during the collaboration meetings of our team.

\section{References}

Basko, M. M., \& Sunyaev, R. A. 1976, MNRAS, 175, 395

Becker, P. A., \& Wolff, M. T. 2007, ApJ, 654, 435

Becker, P., Klochkov, D., Schoenherr, G., et al. 2012, A\&A, in press, DOI: $10.1051 / 0004-6361 / 201219065$

Farinelli, R., Ceccobello, C., Romano, P., \& Titarchuk, L. 2012, A\&A, 538, A67 Isenberg, M., Lamb, D. Q., \& Wang, J. C. L. 1998, ApJ, 493, 154

Klochkov, D., Staubert, R., Santangelo, A., Rothschild, R. E., \& Ferrigno, C. 2011, A\&A, 532, A126

Lebrun, F., Leray, J. P., Lavocat, P., et al. 2003, A\&A, 411, L141

Lund, N., Budtz-Jørgensen, C., Westergaard, N. J., et al. 2003, A\&A, 411, L231

Manousakis, A., Beckmann, V., Bianchin, V., et al. 2008, ATEL, 1613

Mason, K. O., Murdin, P. G., Parkes, G. E., \& Visvanathan, N. 1978, MNRAS, 184,45

McClintock, J. E., Nugent, J. J., Li, F. K., \& Rappaport, S. A. 1977, ApJ, 216, L15

Parkes, G. E., Murdin, P. G., \& Mason, K. O. 1980, MNRAS, 190, 537

Priedhorsky, W. C., \& Terrell, J. 1983, ApJ, 273, 709

Protassov, R., van Dyk, D. A., Connors, A., Kashyap, V. L., \& Siemiginowska, A. 2002, ApJ, 571, 545

Staubert, R., Shakura, N. I., Postnov, K., et al. 2007, A\&A, 465, L25

Trümper, J., Pietsch, W., Reppin, C., et al. 1978, ApJ, 219, L105

Tsygankov, S. S., Lutovinov, A. A., Churazov, E. M., \& Sunyaev, R. A. 2007, Astron. Lett., 33, 368

Tsygankov, S. S., Lutovinov, A. A., \& Serber, A. V. 2010, MNRAS, 401, 1628 Ubertini, P., Lebrun, F., Di Cocco, G., et al. 2003, A\&A, 411, L131

Vedrenne, G., Roques, J.-P., Schönfelder, V., et al. 2003, A\&A, 411, L63

Winkler, C., Courvoisier, T. J.-L., Di Cocco, G., et al. 2003, A\&A, 411, L1

Yamamoto, T., Sugizaki, M., Mihara, T., et al. 2011, PASJ, 63, 751

Yamamoto, T., Tomida, H., Mihara, T., et al. 2012, ATEL, 3856 